\title{
Resource Management in An Integrated Optical Network
}

\author{
Kazem Sohraby, Senior Member, IEEE, Zhensheng Zhang, Senior Member, IEEE, Xiaowen Chu, Member, IEEE, \\ and Bo Li, Senior Member, IEEE
}

\begin{abstract}
We propose a novel integrated optical network switching architecture. The proposal offers an approach to signaling for the purpose of transport on an all-optical network of optical and nonoptical legacy network traffic. In order to provide effective end-to-end control and efficient transport services, new signaling and control techniques are required. Standard organizations such as Optical Interworking Forum (OIF) and Internet Engineering Task Force have developed interface methods between client and transport networks, as well as signaling processes for resource allocation (Benzamin, 2001). We propose a network controller, which implements interfaces for such integration in the intermediate future, as well as provides a feasible path for the long-term objective of all optical networking. Performance and capacity issues for these systems introduce new dimensions to the existing set of networking problems, since optical paths can now be set up in real-time. There are two main contributions in this paper: 1) functional composition of a network controller, which translates legacy signaling to optical connection signaling and path establishment and 2) determining when to issue an optical connection request based on the current network conditions such as link utilization, so that the integrated optical network can operate efficiently. Analytical approximations, as well as simulation results for call blocking performance are also presented.
\end{abstract}

Index Terms-Control, networking, optical networks, performance, signaling.

\section{INTRODUCTION}

$\mathbf{O}$ VER THE LAST decade, technologies related to optical networking, especially the dense wavelength division multiplexing (DWDM), have made significant progress. Many DWDM based systems have been deployed to satisfy the ever increasing Internet traffic demand. However, a large number of legacy networks such as ATM, frame relay, SONET/SDH networks are already in service and should be supported. It is, therefore, critical to efficiently integrate legacy systems with the emerging optical networks.

Today's networks are an increasingly complex interconnection of customer premises equipment, access and switching

Manuscript received August 12, 2002; revised March 20, 2003.

K. Sohraby was with the Bell Labs, Lucent Technologies, Holmdel, NJ 07733 USA. He is now with the Department of Computer and Computer Engineering, College of Engineering, University of Arkansas, Fayetteville, AR 72701 USA (e-mail: sohraby@uark.edu).

Z. Zhang is with Waterridge Networks, San Diego, CA 92130 USA (e-mail: zzhang@ieee.org).

X. Chu is with the Department of Computer Science, Hong Kong Baptist University, Kowloon, Hong Kong (e-mail: chxw@comp.hkbu.edu.hk).

$\mathrm{B}$. Li is with the Department of Computer Science, The Hong Kong University of Science and Technology, Clear Water Bay, Kowloon, Hong Kong (e-mail: bli@cs.ust.hk).

Digital Object Identifier 10.1109/JSAC.2003.815978 nodes, and core network components. The customer premises equipment interface, multiplexing, switching, optical routing, and transport are performed separately under the control of hubs and routers, multiplexers, local switches, optical add/drop multiplexers (OADMs), optical cross-connects (OXCs), and high-capacity DWDM. Currently, this complex set of equipment along with the combination of electronic and optical networking architectures results in a nonintegrated and often separate control for the user traffic over the electronic and optical networks. In principle, the interaction among electronics and optical devices for control and routing makes it possible to deliver traffic from premises equipment to the network for end-to-end transport. However, there does not exist an integrated, single point of control between electronic and optical domains.

Another requirement for integrated optical network infrastructure is as a direct result of increase of volume of multimedia services on the Internet. With the high growth of the data traffic and multimedia services, utilization of optical transport has grown at an unprecedented rate. Along with the traffic growth, high-capacity electronic edge devices (possibly with optical interfaces) connect to the all-optical transport networks. These developments have fundamentally changed the way in which transport networks are designed and operated. The emerging new applications and services on the optical transport network, such as "bandwidth on demand" and "bandwidth trading" require that the transport network respond dynamically to mostly unpredictable demands. An important conclusion drawn from the new set of applications and services is that bandwidth of the transport network needs to be dynamically controlled and allocated on a real-time basis.

The above requirements indicate an urgent need for integrated optical networks. In this paper, we study several key components in an integrated optical network: a network controller which for the purpose of traffic transport, provides signaling and interface to the legacy systems, optical cross-connects, and switching equipment, see Fig. 1. The controller allocates resources for the incoming requests either for the optical path setup and wavelength establishment, or for establishing connections for the requests on the current optical paths; and performs the functions necessary for provisioning of quality-of-service $(\mathrm{QoS})$ and performance guarantees. A major role of the controller, in addition to signaling and control is to monitor the resources in the network in a way that request for a connection over an existing optical path, or a wavelength setup can be optimally allocated while maintaining the performance expectations. The controllers can be managed and operated by the network operators, or the service providers. 


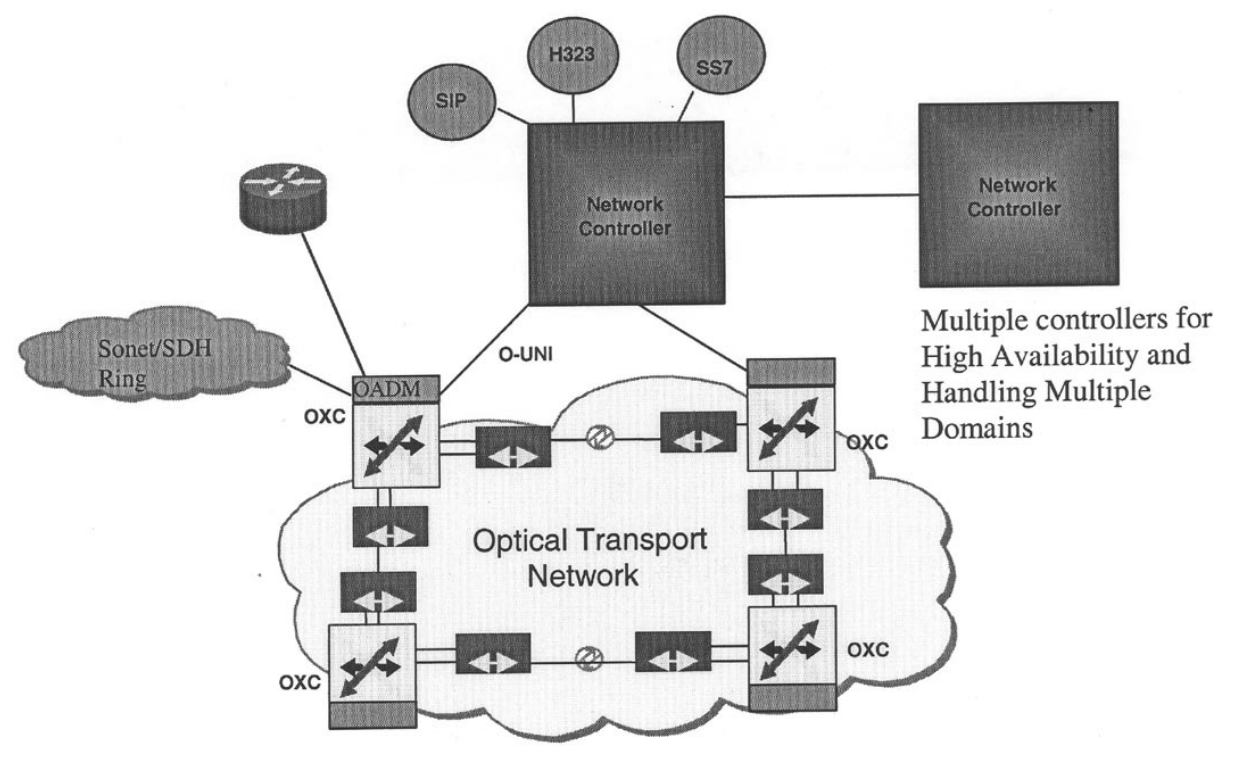

Fig. 1. Integrated optical network.

Impetus to the new switching and signaling method is a new networking paradigm, which calls for the ubiquitous transport of legacy, including optical traffic, and at the same time meet the traffic growth for the Internet and multimedia systems. In this paper, we examine the network controller for the new networking optical paradigm and discuss an integrated end-to-end signaling and control architecture that is independent of the network switching technology. The network controller as proposed in this article is considered as an extension of the existing softswitch product [13], [18]. Softswitch is one of the outcomes of recent advances in converged network technologies and touted as a vehicle for fabric-independent switching and control. Control plane functionalities can be implemented using standards based protocols in order to provide resource management of the bearer channels though circuit and packet switched networks. Heterogeneous sources' traffic such as timedivision multiplexing (TDM) and Internet protocol (IP) can now be transported over ubiquitous IP networks, realizing the economics and technology advantages of a uniform transport. Signaling from various switching end points (e.g., the STP) are terminated at the softswitch; standards-based signaling between Softswitch and the gateway to the transport network is then used to establish connection signaling and resource allocation between those end points. In the current implementations, Internet protocol device control (IPDC), H.248, among other techniques are used for this purpose. In response to the signaling and control from the end points, the softswitch generates the corresponding signaling stimulus at the gateway interface independent of the heterogeneous end points supported by the network.

Network controller for integrated optical network transport provides a similar control and signaling mechanism for integration and transport of legacy systems with the future generation switching and transmission networks in wireless and wireline environments. It is envisioned that the network controller will implement standards such as optical user network interface (OUNI) for client/transport network interface on the one hand, and the legacy systems' signaling on the other. Furthermore, it encompasses signaling procedures such as generalized multiprotocol label switching (GMPLS), which is touted for optical path provisioning and resource allocation.

In optical networks, optical connections or lightpaths should be setup or released in real-time. This enables service providers to order lightpaths dynamically based on demand and network conditions such as link utilization. This is different from some of the legacy networks such as SONET, where it may take weeks or even months to order an $\mathrm{OC} 3$ or OC12 connection. Given that the lightpath provision time is very short, one question the service providers will ask is when to request new lightpaths so that the overall network performance is optimized while the network operational expense is minimized.

In our proposed network controller, there are two main functions:

- integration of the legacy switching and signaling in the optical networks;

- dynamic or real-time allocation/request of lightpaths.

Among the challenges to meet these requirements are: closer examination of the ways that signaling, switching, and transport systems should be planned, designed, and managed in order to provide integrated signaling and control between end user electronics devices and their optical counterpart. A formidable challenge is the performance issues of the network controller, some of which are addressed in this paper.

The rest of the paper is organized as follows. In Section II, we will describe the network controller in detail. Performance analysis of the network controller is provided in Section III; both transient and static cases are considered, respectively. We conclude the paper in Section IV.

\section{NETWORK CONTROLLER}

Before components of the network controller are described, a brief review of the current Internet Engineering Task Force (IETF) standardization effort on GMPLS [15], [16] and OUNI will be given below. As it becomes clear from this discussion, 
the functionalities of the GMPLS and OUNI can be implemented in our proposed network controller.

GMPLS is being considered as the standardized common control plane, which is an essential part of the open and interoperable optical networks. It will be used to dynamically provision resources and to provide network survivability using protection and restoration techniques in data and transmission networks that consist of elements such as routers, switches, DWDM systems, add-drop multiplexers (ADMs), photonic cross-connects (PXCs), optical cross-connects (OXCs), etc. It extends MPLS to encompass time division (e.g., SDH/SONET, PDH, G.709), wavelength (lambdas), and spatial switching. The main focus of GMPLS is on the control plane of various layers since each of them can use physically diverse data or forwarding planes, and it separates control and the forwarding planes. It also decomposes control plane in two parts 1) the signaling plane consisting of the signaling protocols and 2) the routing plane consisting the routing protocols. GMPLS supports the following five interfaces: 1) packet switch capable (PSC); 2) layer-2 switch capable (L2SC); 3) time-division multiplex capable (TDM); 4) lambda switch capable (LSC); and 5) fiber-switch capable (FSC) interfaces. PSC recognizes packet boundaries and forward data based on the content of the packet header. L2SC recognizes frame/cell boundaries and can forward data based on the content of the frame/cell header. TDM forward data based on the assigned time slot. LSC forward data based on the wavelength on which the data is received. FSC forward data based on a position of the data in the physical space. A circuit can be established only between, or through, interfaces of the same type. Depending on the particular technology being used for each interface, different circuit names can be used, e.g., SDH circuit, optical trail, light path, etc. In the context of GMPLS all these circuits are referenced by a common name: label switched path (LSP). A node can support only one type of interface or a combination of these five interfaces, such as shown in Fig. 3.

The OUNI standard provides interface to the optical network or an optical sub-network whereby end devices such as IP routers or SONET add/drop multiplexers, can dynamically request bandwidth. In this case, a signaling channel is required between clients and the optical network control plane to support neighbor and service discovery, address registration, reachability, and provisioning. This interface also specifies the way intelligent optical networks interface with client networks to enable services such as bandwidth-on-demand, point-and-click provisioning and optical virtual private networks (OVPN). In UNI 1.0, an implementation agreement approved by OIF, defines the set of services, the signaling protocols used to invoke the services, the mechanisms used to transport signaling messages, and the auto-discovery procedures that aid in signaling. It is scoped to allow an early implementation based on reusing existing signaling protocols and auto-discovery mechanisms, along with the current and newly available technologies and capabilities in vendor equipment. It should be noted that only signaling for service invocation is within the scope of UNI 1.0. Routing, reachability, and address resolution protocols are outside the scope. At this time, the UNI 1.0 specification focuses on SONET/SDH connection services.

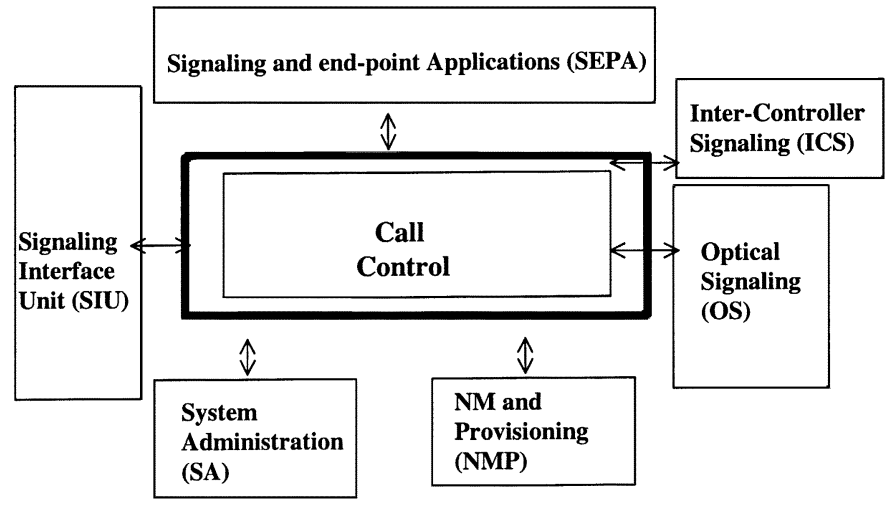

Fig. 2. Functional block diagram of the network controller.

\section{A. Components of the Network Controller}

In the new networking paradigm, network controller plays a critical role and may be viewed as an early implementation of a GMPLS and OUNI controller. Its functions, span from signaling interface to the legacy systems, to the optical devices, as well as interface to future end points. Furthermore, the controller is responsible for lightpath/wavelength request/allocation based on the "direct" incoming requests for optical channels (e.g., "dialed" OC-1, OC-3, etc.), or "indirect" requests for connection establishment over an existing optical path.

The functionality of the network controller depends on the model in which optical networks are operating. Currently, there are two models: overlay, and peer-to-peer [14]. In the overlay model, the network controller requests new lightpaths to be setup or released, but is not involved in actual lightpath routing and setup in the optical network. This model hides the details of the underlying optical network from the end users. Edge devices communicate with optical domain elements through OUNI. In the future peer-to-peer networks, the network controller will be used as peers to the transport nodes in the optical network and will be responsible for lightpath setup.

In this paper, we consider the overlay model in more detail. In this case, the network controller does not setup lightpath in the optical networks.

Fig. 2 shows a functional block diagram of the network controller. It includes signaling interface unit (SIU), signaling and end-point application (SEPA), optical signaling (OS), network management and provisioning (NMP), system administration (SA), and intercontroller signaling (ICS) modules. Each of these modules couples to the call control (CC), which performs processing and control, such as handling the direct and indirect requests for bandwidth allocation. The internal physical interaction among interconnected modules is over a high-speed media with APIs. Specific function of each module is as follows:

1) Signaling Interface Unit (SIU): Interacts with the external legacy signaling systems and end points such as SS7, H323, and SIP. Requests from legacy systems are received at this element; which are forwarded to the CC, and either result in determining an existing optical path to grant the request, or in the need to establish a new optical path, which accommodates the request. In the former case, based on the requested QoS, a decision is made as to 
whether accept or deny the call, and if denied, whether another optical path should be tried. The initial screening of the request and determination of availability of existing optical paths and resources is performed in conjunction with the CC module. In the latter case, when there is a need to establish new optical paths in order to accept the connection, intermodule signaling between $\mathrm{CC}$ and SEPA commences. In this case, a GMPLS signaling message for optical path setup is initiated through SEPA. Specific functions of SIU with respect to the external systems' interfaces, such as ISUP automatic congestion control (ACC), depends on the implementation of the network controller. For example, some functions may be distributed across both CC and the SIU modules (e.g., ISUP ACC), while others may be exclusively performed at the SIU (e.g., SS7 stimulus/response signal composition and STP interaction). For ISDN end points, the D-channel terminates on the SIU. SIU interacts with the CC for call assignment, congestion, overload, and resource allocation.

2) Network Management and Provisioning (NMP): Collects network and equipment initiated alarms, and provides command processing platform for provisioning and reconfiguration of the various modules in the network controller. The controller supports generic interfaces, such as SNMP, for network management and interacts with operator and craft terminals and billing systems for collection of call records, and with the intermediate management systems for status, and alarm information. It downloads updates to the various modules in the controller, and interacts with all modules for reporting and data collection.

3) System Administration (SA): Operator interface for administration and maintenance, such as craft interface terminal (CIT) for manual provisioning, and for intervention such as for setting threshold values for $\mathrm{CC}$, call gap parameters, and protection switching are through the SA module. Through a published open interface, system administration is performed remotely from a centralized management system (e.g., Network Operations Center).

4) Signaling and End-Point Applications (SEPA): This module is home to the electrical and optical transport routing algorithms and resource allocation in conjunction with the OXC controller and routing processors. Elements of GMPLS for optical path set up is located in this module. For example, SEPA contains edge routers' interconnection information, which enables the network controller to either route connections over the existing optical paths, or request establishment of new paths between the edge devices. SEPA sends requests for initiating OUNI messages using the optical signaling module (OS) toward the transport network for optical path set up and reconfiguration for the requests. It also contains configuration information for multidomain optical networks; this module interacts with the external databases and proxies (such as gate-keepers) for translation and for determining network end point addresses, as well as for route determination. In case alarms indicate an optical channel failure, which may require restoration, alternate routing information for the backup optical path or for connection is provided by SEPA to the CC, and with the NMP for record updates. Specific end-user applications, such as intelligent network (IN) and end-user programming interfaces, are also at SEPA.

5) Call Control (CC): This module is the heart of network controller. It is responsible for signaling, call processing from both the legacy systems and the optical transport network, as well as optical path initiation upon receiving connection requests from SIU. Therefore, this module processes incoming signaling messages for call setup and control, either independently using the existing optical paths for which routing information is stored in the SEPA, or by requesting new optical paths. To achieve the tasks of routing, number translation, and signaling, $\mathrm{CC}$ interacts though SEPA with the external databases, proxies, and gatekeepers. CC supports number translation and mapping of call requests onto optical paths. For example, a connection request from the SS7 SIU [e.g., initial address message (IAM)] that requires optical path set up, is first processed at the $\mathrm{CC}$ and if it is determined that there is no existing optical paths to accommodate the request, it forward request for new path establishment to the SEPA, and subsequently uses OSI for the OUNI messaging toward the transport network. Preliminary estimates indicate that each controller domain can support a large number of optical equipment.

6) Optical Signaling (OS): It implements OUNI and in order to determine the available resources in the network, it interacts with the OXC, and other optical transport units. It receives requests from $\mathrm{CC}$ for new optical path setup, and requests or determines a suitable optical path based on routing constraints and the required QoS in conjunction with SEPA and CC. Alarms and status information from the optical transport are received by the OS and are forwarded to the CC, SEPA, and NMP for restoration signaling, admission control, and QoS monitoring.

7) Intercontroller Signaling (ICS): For multidomain networks involving multiple network controllers, ICS is used for signaling exchange among the end points and the gateways.

A network controller platform consists of multiple modules each for performing signaling, call processing, and others in order to support a variety of protocols, network elements, and element types. The number of modules of each type in a controller platform depends on the required capacity and performance determined by engineering guidelines.

In summary, the independence of signaling and transport which is fundamental to the new Network Controller concept, supports a wide variety of heterogeneous end points and can effectively utilize OUNI, and remain consistent and in agreement with the GMPLS standard. As the functional elements demonstrate, with this network controller communications methods between the OS and external legacy systems remain unchanged. It should be noted that without the Network Controller, the interface between legacy systems and the optical 
network, such as through the optical cross connects, would require implementation of new interfaces, such as OUNI on the legacy systems. However, using the controller, legacy systems would use their existing protocols (terminated on the network controller), while OUNI is only implemented at the network controller, with appropriate interfaces to the optical transport. Capabilities of the network controller can now be extended to the service providers for integrated optical network transport, in a much broader sense. For example, a call connection from an end point, with a required QoS, can now be mapped to an optical path between Ingress and Egress under control of the service provider, through SEPA and GMPLS signaling exchange using service provider's supported criteria. The controller monitors various optical channels and incorporates the information into the call admission control.

As mentioned before, in the overlay model, the network controller (through the OS module) requests a lightpath from the optical network using the OUNI signaling. The network controller does not know the detailed configuration of the optical network. However, in the peer-to-peer optical networks, the network controller can be configured to become a peer to the optical network nodes and, hence, will be responsible for lightpath setup using GMPLS [15], [16]. The network controller's SEPA module has the configuration information about the optical network.

\section{Resource Allocation Using Network CONTROLleR}

The set of performance issues encompassed in the design of the network controller covers issues of QoS of traffic that is carried by the optical wavelength (particularly for the "indirect" requests), availability of the wavelength when requests for an optical channel arrives at the controller (i.e., for the "direct" requests) and performance of the controller itself. Network controller performance and in particular its capacity, overload, and congestion control mechanisms, are outside the scope of this paper. The issue discussed in the following, concerns when to request lightpaths and/or allocation of optical channels in response to requests for bandwidth.

It is assumed that the request message for a given bandwidth upon arrival at the network controller carries information about the amount of bandwidth that is to be requested. This can be determined from implied information in the connection request, or the connection type information that is expected at the time. In an alternate approach, the traffic on the incoming links to the gateway at the optical network may be monitored and based on the results of monitoring, bandwidth adjustment can take place, e.g., as proposed in [9].

Network controller handles variety of network trunk types. Among them are: 1) ATM formatted cells on SONET and nonSONET optical links, as well as ATM traffic from DS1, DS3, and higher speed electronic or optical trunks, which terminate at the switch and should be routed through the optical channels; 2) IP packets from routers and Internet edge devices, on optical or electronic trunks; 3) SONET/SDH formatted optical links; 4) DWDM consisting of multiple wavelengths that should be treated as is and not demultiplexed, but may be transported over higher capacity optical links; and 5) TDM trunks (in the form

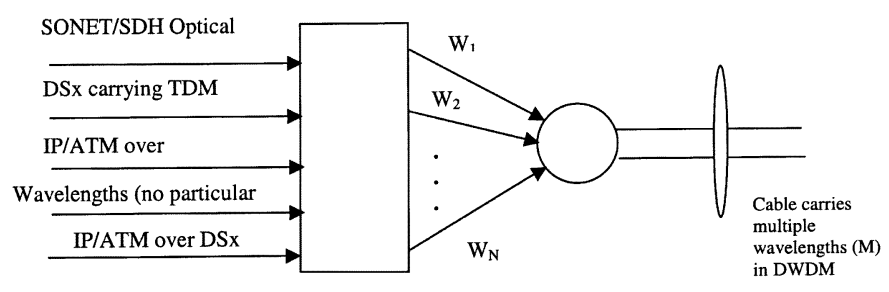

Fig. 3. Network controller controls different trunk types.

of DSx or tributaries on SONET/SDH), such as from electronic circuit switches, which should be separated at the demultiplexer. In this paper, these five categories are assumed to form the major network trunk types that are to be considered in the context of resource allocation at the network controller. As shown in Fig. 3, traffic from these trunks are allocated to wavelengths on the optical fiber. For ease of discussion, the case of resource allocation on optical links across a single source-destination node is considered. The general case of a network consisting of several tandem wavelengths will be considered in the future.

Resource allocation by the network controller is studied from two different points of view: transient and static. In the transient case, optical connections or lightpaths can be requested in real-time. While in the static case, it is assumed that all $M$ available lightpaths have already been allocated. In both cases, call blocking probabilities are studied.

The reasons we separate the two cases are follows: In future optical networks, optical connections or lightpaths can be setup in real-time using the GMPLS control plane. Since the lightpath provision time is very short, network planning will be different from the process that is currently used in traditional networks. In traditional networks, a connection such as DS3 or OC12 takes weeks or even months to be acquired. Therefore, orders for such connections must be planned several months in advance. One should take advantage of the fast provisioning process of the optical networks and request lightpaths accordingly. That is, there is no need to order lightpaths far ahead of time. The question is when to request new lightpaths so that network performance is optimized while the network operation cost including expenses for lightpaths is minimized? Therefore, in the transient case, we assume that lightpaths are provisioned in real-time until all $M$ available lightpaths are utilized. Since lightpaths provisioning time is short and demands for bandwidth are random, one may decide not to request lightpaths too early in order to save cost. In the transient case, we therefore attempt to answer the question: when to request a new lightpath?

When all $M$ available lightpaths are already provisioned in the network, i.e., the static case, we are interested in the call blocking performance.

\section{A. Performance Analysis in the Transient Case}

In this section, we consider the case shown in Fig. 3, assuming a single hop. Furthermore, it is assumed that the number of available lightpaths between nodes changes from time to time and is limited by the number of wavelengths supported by each fiber and the total number of fibers between the two nodes. The access node is virtually a multiplexer in which the outgoing link capacity can be dynamically adjusted. 


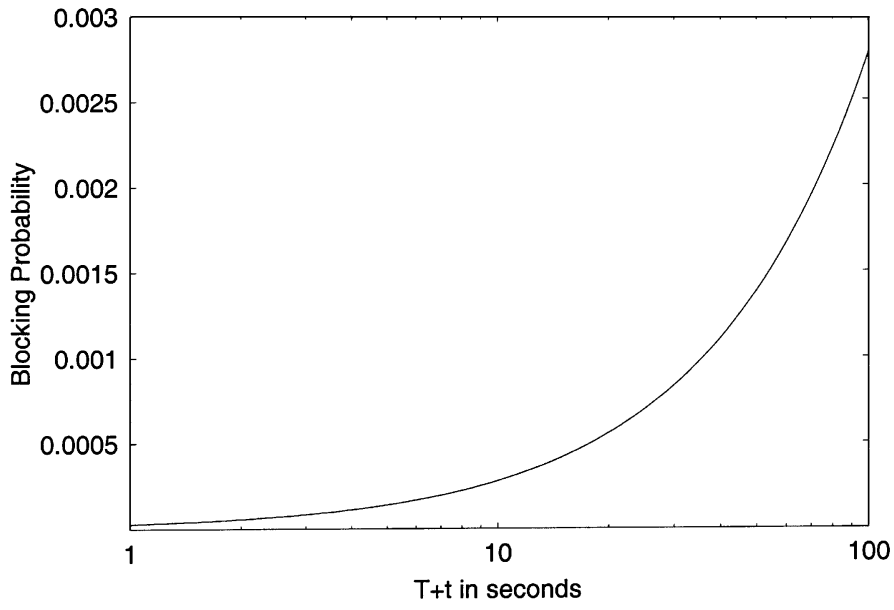

Fig. 4. Call blocking probability versus $T+t$.

Assume that the capacity of the lightpath is OC-192, and that there are $\mathrm{N}$ types of traffic or calls. The bandwidth required by each type, either a constant or variable bit rate with known peak and average bit rates, is assumed to be a fraction of the total lightpath capacity. Connection requests arrive at the system randomly and lightpaths can be requested and released in a very short period of time. When a new connection request arrives, if the bandwidth required by the new call is less than the remaining capacity of a lightpath, the call request is accepted, otherwise, the request is blocked. One approach to reduce the blocking probability is to preprovision the lightpath from the optical network. The question is how long in advance to provision a lightpath? When the number of lightpaths reaches its limit, the blocking probability will be analyzed in Section III-B in the static case. In the following, we study three models based on the traffic characteristics.

Model 1: We consider the simplest case first: The bandwidth required for each call is an OC-192, which is the same as the lightpath's total capacity. Initially, it is assumed that a lightpath is preprovisioned. If a call request arrives, the provisioned lightpath is assigned to the incoming call. We assume that it takes $t$ seconds to provision a lightpath. That is, after a request for lightpath provisioning is issued, $t$ seconds later, the lightpath will be ready to be used for an incoming lightpath request. A lightpath will be released after a connection using the lightpath terminates. To prevent blocking, a new lightpath should be provisioned in advance. Lets assume that a new call is assigned to a preprovisioned lightpath, and that $T$ seconds later a request to provision a new lightpath is issued. Here, $T$ is a control parameter and $t$ is usually fixed. The arrival times of calls are assumed to be Poisson distributed with rate $\lambda$, the mean arrival time being $1 / \lambda \mathrm{s}$. The holding time of the calls are exponentially distributed with rate $\mu$, the mean holding time being $1 / \mu \mathrm{s}$. A call is blocked if it arrives within the time period of $(0, T+t)$. Therefore, the blocking probability, $P_{b}$, is given by $P_{b}=1-e^{-\lambda(T+t)}$.

For the mean arrival time of $1 / \lambda=10 \mathrm{~h}$, Fig. 4 plots the connection blocking probability in the transient case versus the time period $T+t$ (in seconds). As shown in Fig. 4, if the sum of the time to wait and the time to provision the lightpath, $T+t$, is below $100 \mathrm{~s}$, the call probability is only $0.3 \%$.
Suppose that the penalty cost for a blocked call is $C$ and the penalty for not using the provisioned lightpath is $D$, the average penalty cost is, therefore

$$
C\left(1-e^{-\lambda(T+t)}\right)+D\left(\frac{1}{\lambda}-(T+t)\right) .
$$

One objective is to minimize the total penalty cost given that the blocking probability is below a given small value. The problem can be formulated as follows:

$$
\begin{array}{ll}
\text { minimize } & C\left(1-e^{-\lambda(T+t)}\right)+D\left(\frac{1}{\lambda}-(T+t)\right) \\
\text { subject to } & P_{b}=1-e^{-\lambda(T+t)}<=\varepsilon
\end{array}
$$

where $\varepsilon$ is the objective connection blocking probability.

The optimal value of the time interval $T$ (when to request to provision a new lightpath) can be easily obtained and is given as follows: if $\lambda C \leq D$, the optimal value of $T$ in (1), $T^{\mathrm{opt}}=$ $-t-\ln (1-\varepsilon) / \lambda$ and, otherwise, $T^{\mathrm{opt}}=0$. This indicates that for large $C(C>D / \lambda)$, the optimal value of $T$ is zero, as expected. If $C$ is relatively small, the larger the value of $T$, the smaller the overall penalty, while the constraint in (2) is satisfied.

Model 2: Assume that there are $N$ types of incoming call requests. The bandwidth required by call type $i$ is $b_{i}$, $i=1,2, \ldots, N$. For example, $b_{i}$ could be an OC-1, OC-3, OC-12, OC-48, or OC-192. The capacity of a lightpath is assumed to be OC-192. Initially, it is assumed that a lightpath is already provisioned. When a new call request arrives, the new call is assigned to the provisioned lightpath. In normal operation, every time a new call is accepted, the remaining capacities of the lightpaths are computed. If the largest remaining capacity is less than $Q$, a predefined threshold, a new lightpath provisioning request will be issued. Again it will take $t$ seconds to setup the lightpath. If the bandwidth required by the new call is less than the remaining capacity of the lightpaths, the new call is accepted and assigned to an existing lightpath; otherwise, the new call is blocked. Note that in order to accept as many calls as possible, we assign the new call to the lightpath whose remaining capacity is the smallest among those whose remaining capacities are greater than the required bandwidth. We assume that a call is entirely allocated on the same lightpath. That is a new call will not be split into "sub" calls. A lightpath is released after all calls on the lightpath terminate. To increase link utilization, the existing calls on a lightpath can be rearranged after some calls depart, in order to open up capacity for new incoming requests. For example, if the remaining capacity on lightpath 1 is OC-12 and there is only one OC- 3 call on lightpath 2 , one may move the OC- 3 to lightpath 1 and release lightpath 2 .

The call blocking probability depends on the value of the threshold $Q$. For example, if the value of $Q$ is less than an OC-12, then all the calls which requires an OC-12 or higher will be blocked. The call blocking probability can be derived as follows. Let us assume that the types of calls are arranged in 
an ascending order in terms of the bandwidth requested. That is, the bandwidth required by type $1, b_{1}$, is the smallest and by type $N, b_{N}$ the largest. Assume that there is a $j_{Q}, j_{Q}<N$, such that $b_{j_{Q}-1} \leq Q$ and $b_{j_{Q}}>Q$; or $j_{Q}=N$, when $b_{N} \geq Q$. Then, the call blocking probability, which depends on the value of $Q$, is given by

$$
P_{b}(Q)=1-e^{-\left(\lambda_{j_{Q}}+\lambda_{j_{Q}}+1+\cdots+\lambda_{N}\right)(T+t)} .
$$

Note that some of the lightpaths may not be fully utilized, the bigger the value of $Q$, the lower the lightpath utilization is. On the other hand, the bigger the value of $Q$, the bigger the value of $j_{Q}$ which implies, from (3), the smaller the blocking probability. Similar numerical results of the call blocking as in Fig. 4 can be obtained for a given value of $Q$. Similar optimization problems as in (1) and (2) can also be formulated; $Q$ can be treated as fixed or as a variable control parameter.

It is possible that, after a lightpath provisioning request is issued but the lightpath is not provisioned yet, a new call request arrives and is accepted into one of the existing lightpaths. In this case, a new lightpath provisioning request will not be issued for the latter as a request is still pending.

Model 3: In model 2, we assumed that the bandwidth required by each connection is a constant. However, this assumption may not be valid in the case with the Internet connections. In this model, we assume that each connection uses a variable bite rate (VBR) with an average bit rate, $b_{j}$ and peak rare $p_{j}$. The burst length corresponding to the peak rate is assumed to be generally distributed, that is the distribution of the burst length is arbitrary. Initially, there is one provisioned lightpath. When a new request arrives, it is accepted. In the normal operation, the remaining capacities of the lightpaths are periodically measured (alternatively, they can be measured at the times when there are new arrivals or departures). Let $B_{i}(t)$ be the remaining bandwidth measured at time $(t)$ on lightpath $i$. There are several methods regarding how the measured data can be used in the decision process to accept or reject a request. Let $\overline{B_{i}(t)}$ be the average BW calculated based on a filtering method. For example, $\overline{B_{i}(t)}$ can be determined by: 1) using the bandwidth measured at time $t$, that is $\overline{B_{i}(t)}=B_{i}(t)$ or 2$)$ taking the exponential weighted moving average (EWMA) of the measured remaining bandwidth, that is $\overline{B_{i}(t)}=\eta B_{i}(t)+(1-\eta) \overline{B_{i}(t-1)}$, where $\eta$ is the gain, or 3) sliding window, where $\overline{B_{i}(t)}=\sum_{k=0}^{n-1} B(t-k)$, where $(n)$ is the window size. Since the decision whether a call is accepted is based on the value of the remaining bandwidth at the current time, in this paper, for simplicity we use method 1). When a new call of type $j$ arrives, if there is at least one lightpath whose remaining capacity $\overline{B_{j}(t)}$ is below $\alpha_{j} p_{j}$, $\left(0<\alpha_{j} \leq 1\right)$, the new call is accepted into the system. If there are more than one such lightpaths (whose remaining capacities are below $\alpha_{j} p_{j}$ ), the lightpath with the smallest remaining capacity will be chosen to accommodate the new call. Note that $\alpha_{j}$ is an admission control parameter, where $\alpha_{j}=1$ means that the call is admitted based on its peak rate and $\alpha_{j}=b_{j} / p_{j}$ means that the call is admitted based on its average rate. To increase the lightpaths efficiency, calls can be shifted from one lightpath to another lightpath.

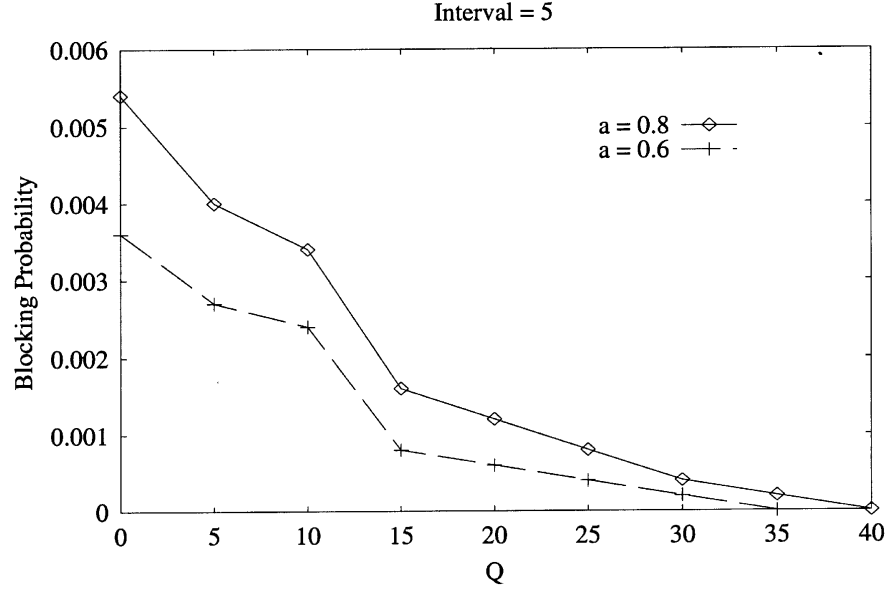

Fig. 5. Call blocking probability versus threshold $Q$.

Recall that the remaining capacities of the lightpaths are measured periodically, if the largest remaining capacity is less than $Q$ (a predefined threshold as in model 2), a new lightpath will be requested. A lightpath is released if all the traffic in the lightpath terminate. The performance of the system depends heavily on the characteristics of the traffic such as peak rate, average rate, mean burst length, etc. Theoretical analysis for this case is difficult, therefore, in the following, we carry out simulation to study the call blocking of the system.

For simplicity, in the simulation, we assume that all traffic sources are identical, that is $\alpha_{j}=\alpha, b_{j}=b$, and $p_{j}=p$ for all $j$. It has been demonstrated in the past that most of the Internet traffic flow durations have a well-known heavy tail property, which can be modeled as a Pareto distribution [17]. Therefore, we assume the call duration to be Pareto distributed. That is, $F(x)=1-(k / x)^{\beta}, x \geq k, 1<\beta>2$. In the period of call duration, the bit rate is assumed to be an ON-OFF process with its peak rate $p$ when it is ON, and zero when it is OFF. It is assumed that the "ON" periods are uniformly distributed, while the "OFF" periods are exponentially distributed. The remaining capacities of the lightpaths are measured periodically with periods as indicated by the interval value stated on each figure. In Figs. 5-8 and in Table I, we make the following assumptions, the unit can be seconds, minutes or hours.

- Call arrival: Exponentially distributed with mean of 5 (in Fig. 8, the mean is 2.5).

- Call holding time: Pareto distributed with average of 1000 (in Figs. 5-8, $\beta=1.5$ and $k=1000 / 3$ ).

- The bandwidth required during each call follows an ON-OFF process: during the ON period, the bandwidth required by the call is its peak rate, while during the OFF period, the bandwidth required by the call is zero.

- On-period time: Uniformly distributed between 5 and 15.

- Off-period time: Exponentially distributed with mean of 5.

- Four classes of traffics are assumed: the peak rates are OC1, OC 3, OC12, and OC48. Total capacity of a lightpath is an OC192.

Simulation Results: Some observations with regards to Figs. 5 and 6. 


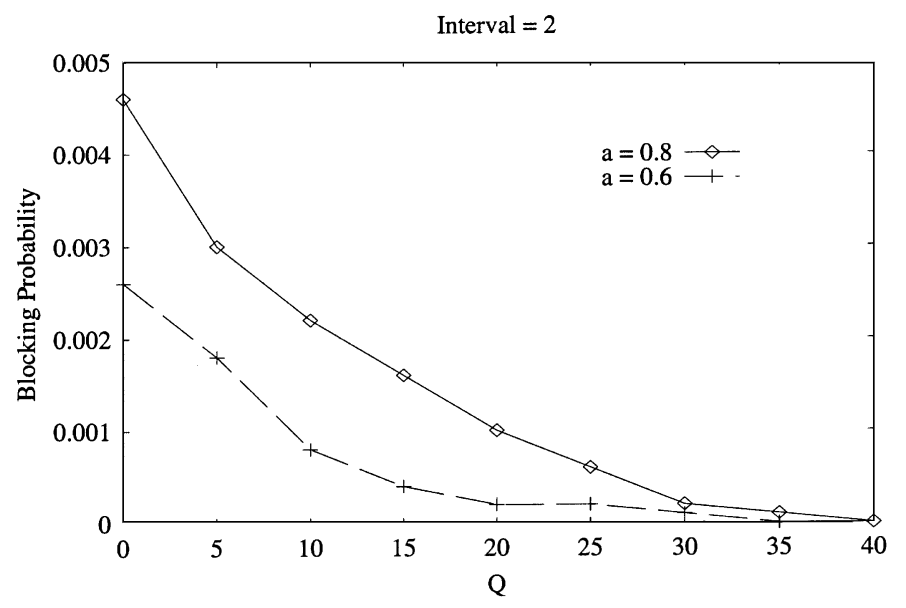

Fig. 6. Call blocking probability versus threshold $Q$.

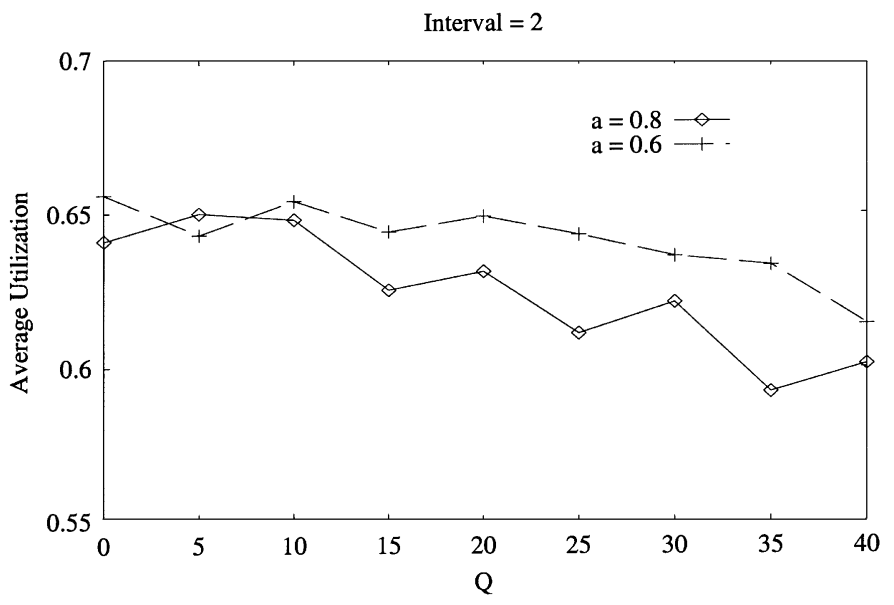

Fig. 7. Average link utilization versus threshold $Q$.

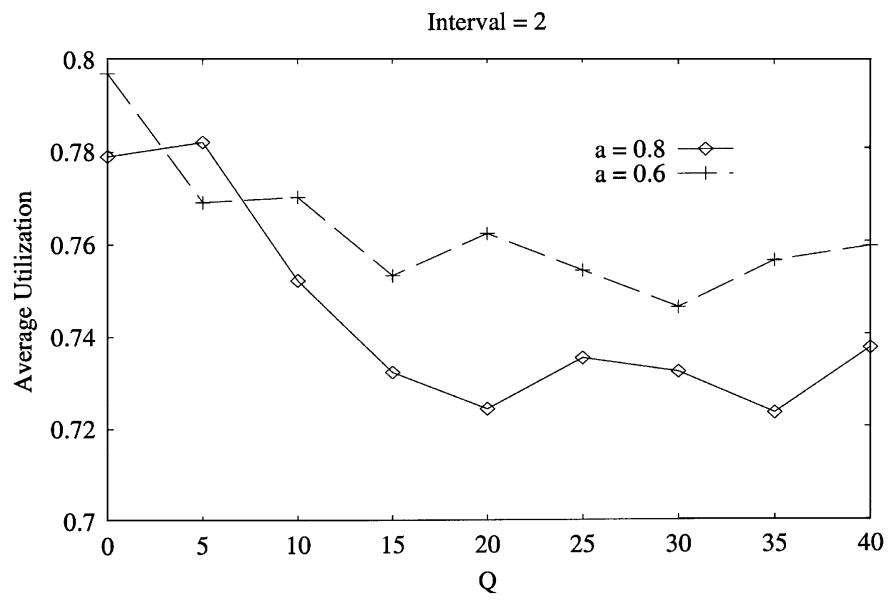

Fig. 8. Average link utilization versus threshold $Q$.

1) When $Q$ is small, the blocking probability decreases dramatically with increasing $Q$. However, when $Q$ reaches a certain value $(\sim>15)$, the decline in blocking (as $Q$ increases) is very small.

2) As expected, the smaller the value of $\alpha$, the smaller the blocking probability since more calls are admitted into the system.

3) The smaller the measurement interval, the smaller the blocking probability as higher total remaining capacity is obtained. But the smaller the measurement interval, the more overhead the system has.

In these simulations, the parameter of call arrival interval time is set to 2.5 in Fig. 8, thus, the total traffic is double the amount in Fig. 7.

From the simulation results, we observe that the average link utilization depends heavily on the traffic load. The average link utilization decreases as the threshold increases since more lightpaths will be provisioned. The relation between the link utilization and the admission parameter $\alpha$ is that the smaller the $\alpha$, the higher the link utilization, as expected since more calls are admitted into the system.

In Table I, we present the blocking probability versus the value of $Q$ for three different values of $\beta$ in the Pareto distribution while still keeping the mean call holding time fixed at 1000 . From Table I, we notice that different $\beta$ values result in different blocking probabilities. When $\beta=1.9$ compared with $\beta=50 / 49$, although the mean call holding time remains the same in the two cases, it is expected that the holding time has longer tail and, therefore, it results in higher probability of blocking.

From the above simulation results, we conclude that how to determine the value of $Q$ depends on performance objective such as call blocking, link utilization, and the traffic characteristics, etc., as expected.

\section{B. Performance Analysis of the Static Case}

In this case, we assume that the maximum number of wavelengths carried by the DWDM on each outgoing link is (M), and unlike the transient case, all $M$ wavelengths have already been preprovisioned. The numerical results, unless otherwise noted, are obtained through simulation. Among the incoming trunks to be controlled by the network controller, a number of wavelengths are set aside as fixed, and do not vary with time, e.g., the end-end connections that are allocated for a given network configuration and are not subject to time-of-day or other dynamics of traffic change. However, another group of such trunks would require time-of-day adjustment, either based on traffic demand, or network reconfiguration requirements. In the latter case, the number of wavelengths allocated varies and of primary interest in the admission control is the time scale of the requests handled at the controller. The demand from the fixed terminations $\left(N_{f}\right)$ should be maintained for an indefinite period of time. Demands from the TDM terminations $\left(N_{t}\right)$, on the other hand, most likely will vary with the time of day because requests are originated by the telecommunications service providers, and providers of circuit switched services. Generally, service providers reconfigure their networks based on traffic engineering rules that reflect demand shifts in the network during the day and try to minimize call blocking and/or maximize revenue, [10].

An important traffic type to be considered here is the "dialed wavelength" through the network controller. These require an entire wavelength, or portion thereof. For example, the dial request can be for an OC-1, OC-3, OC-12, or even OC-192. These may require a short or long period of connection; they may be used for backup of a failed optical link (several hours), or for a highspeed connection (e.g., video), typically for few hours. Due to 
TABLE I

BLOCKING PROBABILITY, INTERVAL $=5, \alpha=0.6$

\begin{tabular}{c|c|c|c|c|c|c|c|c|c}
\hline$\beta$ & $\mathrm{Q}=0$ & $\mathrm{Q}=5$ & $\mathrm{Q}=10$ & $\mathrm{Q}=15$ & $\mathrm{Q}=20$ & $\mathrm{Q}=25$ & $\mathrm{Q}=30$ & $\mathrm{Q}=35$ & $\mathrm{Q}=40$ \\
\hline $\begin{array}{c}1.9 \\
(\mathrm{k}=900 / 1.9)\end{array}$ & $0.44 \%$ & $0.34 \%$ & $0.11 \%$ & $0.08 \%$ & $0.07 \%$ & $0.05 \%$ & $0.04 \%$ & $0.01 \%$ & $0 \%$ \\
\hline $\begin{array}{c}1.5 \\
(\mathrm{k}=1000 / 3)\end{array}$ & $0.36 \%$ & $0.27 \%$ & $0.24 \%$ & $0.08 \%$ & $0.06 \%$ & $0.04 \%$ & $0.02 \%$ & $0 \%$ & $0 \%$ \\
\hline $\begin{array}{c}50 / 49 \\
(\mathrm{k}=20)\end{array}$ & $0.09 \%$ & $0.06 \%$ & $0.05 \%$ & $0.03 \%$ & $0.02 \%$ & $0.01 \%$ & $0 \%$ & $0 \%$ & $0 \%$ \\
\hline
\end{tabular}

TABLE II

PRobability of ReQuest REJects (COMPLETE SHARING)

\begin{tabular}{c|c|c|c|c|c}
\hline$M$ & $\begin{array}{c}\text { Requests/Day } \\
(\text { OC1,OC3,OC12,OC192) }\end{array}$ & OC1 & OC3 & OC12 & OC192 \\
\hline 1 & $400,200,100,50$ & 0.011 & 0.033 & 0.143 & 1.0 \\
\hline 2 & $400,200,100,50$ & 0.007 & 0.022 & 0.093 & 0.910 \\
\hline 16 & $400,200,100,50$ & 0.00 & 0.0001 & 0.0003 & 0.023 \\
\hline
\end{tabular}

the time scale differences between the time-of-day connection requests and the relatively shorter time scale requests for subrate and full-wavelength requests, the admission control problem at the network controller is unique and can be stated as follows.

Given the number of wavelength requests for the fixed, or for the time-of-day reconfigured channels, as well as for subrate and full-rate dialed wavelengths, what is the optimal call admission control at the network controller?

The solution to this problem can be made either dependent or independent of the economies of the transport. In fact, if a short duration connection request is granted, it is entirely possible that, henceforth, due to the unavailability of larger BW channels, the controller is forced to reject a time-of-day wavelength request that is for longer connection duration and, therefore, will likely lose higher revenue. On the other hand, blocking of short duration calls (given the unpredictability of the connection duration) introduces other performance issues. It is important to note that in addition to the requests for the optical wavelengths (e.g., OC-1, OC-3, OC-12, OC-192, etc.), "connection requests" for calls with much smaller bandwidth than the wavelength channel may also arrive at the controller (referred to as indirect requests in this paper).

Resource Allocation Model: In order to analyze the performance of network controller, we adopt a model similar to the traditional resource allocation in circuit switched networks, with some modifications. To begin with, we assume that there are total of (M) DWDM wavelength channels on the optical fiber. Each channel is assumed to be at OC-192 rate (about $10 \mathrm{~Gb} / \mathrm{s}$ ), each of which can accommodate 192 OC-1s, 64 OC-3s, 16 OC-12s, or a combination thereof. For example, when an OC-12 is available on an optical link, it is feasible to accommodate at least four $\mathrm{OC}-3 \mathrm{~s}$, or $12 \mathrm{OC}-1 \mathrm{~s}$ on that link.

We also assume that $\left(N_{R}\right)$ OC-192 channels are set aside (reserved) for fixed network configuration in the reservation pool. These can change over time if necessary in response to wavelength demand, however, its rate of change is assumed to be very small and, thus, infrequent changes to the value of $N_{R}$ is required. The rate of request for the time-of-day changes for the OC-192 channels is assumed to be $\lambda_{d}$ for the nonreserved channels. Each request would correspond to a number of OC-192
TABLE III

DEDICATED WAVELENGTHS FOR OC-192

\begin{tabular}{c|c}
\hline $\mathbf{N}_{\mathbf{R}}$ & Probability of OC192 Requests Rejected \\
\hline 2 & 0.79 \\
\hline 3 & 0.68 \\
\hline 10 & 0.14 \\
\hline 12 & 0.06 \\
\hline 14 & 0.02 \\
\hline 15 & 0.012 \\
\hline 16 & 0.005 \\
\hline
\end{tabular}

channels to be allocated for the duration of the network configuration. Furthermore, it is assumed that partial wavelength (e.g., OC-1, OC-3, or OC-12) requests arrive at the controller at the rate $\lambda_{o 1}, \lambda_{o 3}, \lambda_{o 12}$, respectively, while the rate of requests for full wavelength of OC-192 is $\lambda_{o 192}$. All request arrivals follow a Poisson process (i.e., interarrival times of requests is exponentially distributed), with the specified rates.

The maximum number of wavelengths (i.e., OC-192 channels) available at the controller is $M$, where $M>N_{R}$. Given limited number of wavelengths between the two nodes, a performance measure of interest is the rejection rate (probability of blocking) for BW requests at the controller, and then if possible, to devise an approach to improve those rates. In the following three resource allocation methods: complete sharing, complete partitioning, and partial sharing are discussed and request rejection rates are presented.

- Complete Sharing: In this case, all the channels on the optical cable are shared among the different BWs. There is no reservation/allocation for a particular $\mathrm{BW}$, and no preemption method is adopted. Table II below shows the results of request rejection rates at the controller where the DWDM supports maximum of $M=16$ wavelengths. All 16 wavelengths are shared among the incoming requests for the available capacity on the optical link. All requests are served on a FIFO basis. When $M=1$, even if a single OC- 1 connection is in progress, an incoming request to the controller for an OC-192 channel would be rejected.

Parameters of the model are the rate of request per day for the various OC rates, and the channel holding times. 
TABLE IV

REQUEST REJECTION RATE FOR PARTIAL SHARING

\begin{tabular}{c|c|c|c|c|c}
\hline $\begin{array}{c}\text { Shared } \\
\text { Wavelengths }\end{array}$ & $\begin{array}{c}\text { Reserved } \\
\text { Wavelengths } \\
\left(\mathrm{N}_{\mathrm{R}}\right)\end{array}$ & OC-1 & OC-3 & OC-12 & OC-192 \\
\hline 16 & 0 & 0. & 0.0001 & 0.0003 & 0.0229 \\
\hline 14 & 2 & 0. & 0.0001 & 0.0006 & 0.0213 \\
\hline 2 & 14 & 0.0003 & 0.0011 & 0.0048 & 0.0191 \\
\hline
\end{tabular}

The average for exponentially distributed channel holding times are: $1 \mathrm{~h}$ for OC-1, $2 \mathrm{~h}$ for OC-3, $2 \mathrm{~h}$ for OC-12, and $4 \mathrm{~h}$ for OC-192. As this table shows, as the number of wavelengths $M$ increases, the probability of request rejection for all channel types decreases. In particular, in the case of $M=1$ (one DWDM channel only), all OC-192 requests are rejected (i.e., probability of OC192 blocking is one in Table I). Other requests may also be rejected, but with a smaller probability. As expected, the OC-1 requests are rejected at the smallest rate among all others, since at the lowest BW, it is more likely to find available capacity to launch the request. At $M=16$, virtually all requests are granted (with a small rejection rate for OC-192).

- Complete Partitioning: In this case, a number of wavelength channels are allocated to the various BW requests on a dedicated basis. In the following examples in Table II results of several cases where $\left(N_{R}\right)$ wavelengths are assumed to be dedicated to the OC-192 channels are shown. The results in this table can be contrasted with the complete sharing case. As observed from these results, when requests for OC-192 arrive at the network controller, the likelihood of the request being accepted is higher in complete partitioning than in the complete sharing. The $\left(N_{R}\right)$ wavelengths are not shared with the lower rate channels. Request rejection rates in this case can be obtained from the Erlang-B formula, [12]

$$
P_{B}=\frac{\rho^{N}}{N ! \sum_{i=0}^{N}\left(\frac{\rho^{i}}{i !}\right)}
$$

where $\rho=\lambda_{o 192} / \mu_{o 192}$ is the ratio of arrival requests over channel holding rate for the OC-192.

As Table III shows, with $M=N_{R}=2$ the request rejection rate for OC-192 in complete partitioning reduces to $79 \%$ from $91 \%$ in the complete sharing. Similar improvements are observed for different number of dedicated DWDM channels.

- Partial Sharing: This refers to a combination of partitioning and sharing. A number $\left(N_{R}\right)$ of wavelengths are kept in a reservation pool say for the OC-192 requests (i.e., no other OC rates can utilize these channels). The remaining wavelengths are assumed shared among all incoming requests. Upon receiving a request for OC-192 at the controller, first the reserved pool of OC-192 is tried. If the request cannot be accommodated at the reserved pool, the controller examines the shared pool. As expected, for the same total number of wavelengths in the DWDM pool, rejection rate for OC-192 requests improves, while the rejection rates of lower rate requests increase. As results in Table IV show, the gain obtained for OC-192 rejection rates through reservation, can be significant. Also, as Table Table IV shows, with partial sharing, as the number of shared wavelengths decline, there is an increase in the rejection rate of the $\mathrm{OC}-1, \mathrm{OC}-3$, and $\mathrm{OC}-12$ requests, even so insignificantly.

\section{CONCLUSION}

The integrated optical transport, as a new vehicle for signaling, switching, and control on the path to an all optical networking, calls for new techniques that are similar in some ways, and quite different in others, from the current ones, in terms of resource allocation. In this paper, we propose a network controller which, as the main element of integrated optical networks, encompasses intelligent control, and performs signaling across legacy and optical networks. While its implementation remains consistent with the standards for OUNI and GMPLS, the network controller extends control of the transport network and of the individual connections to the service providers and end users. Realization of the network controller accelerates service introduction and network expansion. As a result, service providers will be able to dynamically allocate network resources in response to mostly unpredictable demands. We also address a new problem in optical networks: when to provision new lightpaths based on the current network conditions? Three resource allocation methods are also considered, and their performance compared. The performance models described here are for a single link case. We are currently considering the system performance for multiple link case.

\section{REFERENCES}

[1] Internet Protocol Device Control, Revision 0.17, 2000

[2] J. T. Moy, OSPF Anatomy of an Internet Routing Protocol. Reading, MA: Addison-Wesley, 1998.

[3] K. Sohraby, M. Fatehi, V. Lawrence, and M. Wilson, "Network architecture for an all-optical internet," in NFOEC 2000, Denver, CO.

[4] "User Network Interface (UNI) 1.0 Signaling Specification," Optical Interworking Forum, Fremont, CA, Contribution OIF2000.125.7, 2001.

[5] D. Benzamin, R. Trudel, S. Shew, and E. Kus, "Optical services over the intelligent optical network," IEEE Commun. Mag., pp. 73-78, Sept. 2001.

[6] U. Schoen et al., "Convergence between public switching and the internet," IEEE Commun. Mag., pp. 50-65, Jan. 1998.

[7] C. Low, "Integrating communications services," IEEE Commun. Mag., pp. 164-169, June 1997.

[8] D. Jeske, B. Samadi, K. Sohraby, Y.-T. Wang, and Q. Zhang, "QoS with an edge-based call admission control in IP networks," presented at the Proc. Networking, Pisa, Italy, May 2002. 
[9] K. Sohraby and M. Fatehi, "Digital container: A mechanism for heterogeneous traffic transport over an all-optical network," Opt. Networks, vol. 2, no. 3, pp. 45-57, May/June 2001.

[10] G. R. Ash, Dynamic Routing in Telecommunications Networks. New York: McGraw-Hill, 1997.

[11] K. Sohraby and K. Ryan, "An architecture for signaling, switching, and control in optical networks," presented at the 6th World Multiconference on Systematics, Cybernetics, and Informatics (SCI), Orlando, FL, July 2002.

[12] J. Bellamy, Digital Telephony, 2nd ed. New York: Wiley, 1990.

[13] [Online]. Available: http://www.lucent.com

[14] Z. Zhang and X. Liu, "Network models for IP over optical networks," Opt. Network Mag., vol. 1, no. 4, pp. 12-14, Oct. 2000.

[15] "Generalized Multi-Protocol Label Switching (GMPLS) Architecture,", draft-ietf-ccamp-gmpls-architecture-03.txt, work in progress.

[16] Z. Zhang, D. Guo, L. Zhang, and J. Fu, "Lightpath routings in intelligent optical networks," IEEE Network, vol. 15, pp. 28-35, July/Aug. 2001.

[17] K. Thompson et al., "Wide area internet traffic patterns and characteristics," IEEE Network, vol. 11, pp. 10-23, Nov. 1997.

[18] R. A. Lakshmi, "The lucent technologies softswitch: Realizing the promise of convergence," Bell Labs Tech. J., vol. V24, no. 2, Apr.-June 1999.

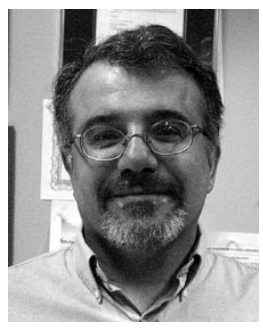

Kazem Sohraby (S'74-M'78-SM'89) received the B.S., M.S., and Ph.D. degrees in electrical engineering and the M.B.A. from the Wharton School, University of Pennsylvania, Philadephia.

$\mathrm{He}$ is a Professor and Head of the Computer Science and Computer Engineering Department, College of Engineering, University of Arkansas, Fayetteville. Prior to that, he was with Bell Laboratories, Holmdel, NJ. His areas of interest include computer networking, signaling, switching, performance analysis, and traffic theory. He has over 20 applications and granted patents on computer protocols, wireless and optical systems, circuit and packet switching, and on optical Internet. He has several publications, including a book on The Performance and Control of Computer Communications Networks (Boston, MA: 1995).

Dr. Sohraby is a Distinguished Lecturer of the IEEE Communications Society, and serves as its President's representative on the Committee on Communications and Information Policy (CCIP). He served on the Education Committee of the IEEE Communications Society, is on the Editorial Boards of several publications, and served as Reviewer and Panelist with the National Science Foundation, the U.S. Army and the Natural Sciences and Engineering Research Council of Canada.

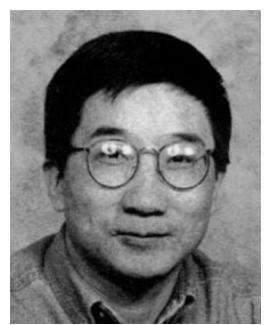

Zhensheng Zhang (M'90-SM'01) received the Ph.D. degree in electrical engineering from the University of California, Los Angeles.

$\mathrm{He}$ is currently with Waterridge Networks, San Diego, CA. He has visited Microsoft Research Asia, Beijing. China. He was with the Department of System Architecture, Sorrento Networks, San Diego, CA, for two years, responsible for designing the next-generation optical metro networks using the GMPLS control framework. Prior to joining Sorrento Networks, he was with Bell Laboratories, Lucent Technologies, Holmdel, NJ, focusing on research and development in ATM/SONET infrastructure and IP over WDM. He has published more than 100 papers in the IEEE Transactions on Networking, the IEEE JOURNAL on SElected areas in Communications, the IEEE TRansactions on COMMUNICATIONS, and key IEEE conferences. His research interests include wireless networks, IP over WDM networks, GMPLS, and peer to peer networks.

Dr. Zhang has served as Guest Editor for the IEEE JouRnAL ON SELECTED AREAS IN COMMUNICATIONS, Special Issue on Overlay Networks, 2003, and the Special Issue on IP over DWDM networks, October 2000, and the Journal of Wireless Networks issue on Multimedia Wireless Networks, August 1996. Currently, he is Editor of IEEE TRANSACTION ON WIRELESS COMMUNICATIONS and is on the Editorial Board of Optical Networks Magazine. He is a recipient of the 1988 Phi Beta Kappa Scholarship Award.

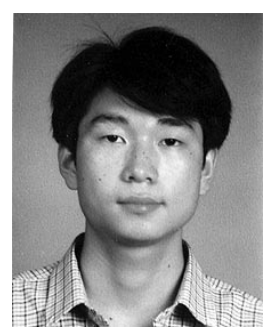

Xiaowen Chu (S'01-M'03) received the B.S. degree in computer science from Tsinghua University, Beijing, China, in 1999 and the Ph.D. degree in computer science from the Hong Kong University of Science and Technology, Kowloon, in 2003.

$\mathrm{He}$ is currently with the Computer Science Department, Hong Kong Baptist University, Kowloon. His current research interests include optical WDM networks, Internet security, and high-performance routing and switching.

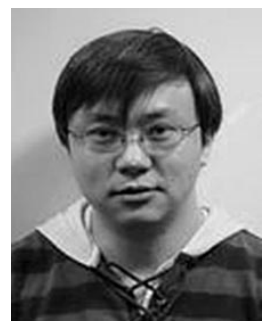

Bo Li (S'89-M'92-SM'99) received the B.S. (summa cu laude) and M.S. degrees in computer science from Tsinghua University, Beijing, China, in 1987 and 1989, respectively, and the Ph.D. degree in computer engineering from the University of Massachusetts, Amherst, in 1993

From 1994 and 1996, he worked on high-performance routers and ATM switches with IBM Networking System Division, Research Triangle Park, NC. Since then, he has been with the Computer Science Department, Hong Kong University of Science and Technology, Kowloon. He is also an Adjunct Researcher at Microsoft Research, Asia. His current research interests are on wavelength-routed networks, video multicast, resource management in wireless cellular systems, web caching, and service overlay networks.

Dr. Li have been fairly active in IEEE and the Association for Computing Machinery (ACM). He has been on the Editorial Boards of the IEEE TRANSACTIONS ON WIRELESS COMMUNICATIONS, the IEEE TRANSACTIONS on Vehicular Technology, the IEEE Journal of Selected AREAS IN Communications-Wireless Communications Series, ACM Mobile Computing and Communications Review (MC2R), Elsevier Ad Hoc Networks, SPIE/Kluwer Optical Networks Magazine (ONM), IEEE JouRNAL OF COMMUNICATIONS AND NETWORKS (JCN). He has served as a Guest Editor for the IEEE Communications Magazine, Special Issue on Active, Programmable, and Mobile Code Networking (April 2000), the IEEE Journal ON SELECTED AREAS IN COMMUNICATIONS, Special Issue on Protocols for Next-Generation Optical WDM Networks (October 2000), an ACM Performance Evaluation Review, Special Issue on Mobile Computing (December 2000), and SPIE/Kluwer Optical Networks Magazine, Special Issue on Wavelength Routed Networks: Architecture, Protocols, and Experiments (January/February 2002). Currently, he is Co-Guest Editing two special issues for the IEEE JouRNAL ON SELECTED AREAS IN COMmUnications, on Recent Advances in Service-Overlay Network, and on Quality of Service Delivery in Variable Topology Networks. In addition, he has been involved in organizing over 30 conferences, especially IEEE INFOCOM since 1996. He is the Co-TPC Chair for IEEE INFOCOM 2004 